\title{
Uncovering the gut microbiota as a reservoir of ST11 hypervirulent KPC-2-producing Klebsiella pneumoniaeUncovering the gut microbiota as a reservoir of ST11 hypervirulent KPC-2-producing Klebsiella pneumoniae
}

Beiwen Zheng ( $\square$ zhengbw@zju.edu.cn )

ZHEJIANG UNIVERSITY https://orcid.org/0000-0002-7690-372X

Hao Xu

Zhejiang University

Tao Lv

Zhejiang University

Lihua Guo

Zhejiang University

Xiao Yu

Zhejiang University

Chen Huang

Zhejiang University

Shuntian Zhang

Zhejiang University

Yunbo Chen

Zhejiang University

Huiming Han

Beihua University

Ping Shen

Zhejiang University

Yonghong Xiao

Zhejiang University

Lanjuan Li

Zhejiang University

Research

Keywords: gut microbiota, hypervirulent, KPC-2, reservoir, genomic characterization 
Posted Date: November 25th, 2019

DOI: https://doi.org/10.21203/rs.2.17648/v1

License: (c) (i) This work is licensed under a Creative Commons Attribution 4.0 International License. Read Full License 


\section{Abstract}

Background: The emergence and spread of ST11 carbapenem-resistant, hypervirulent K. pneumonia (ST11-CR-HvKP) in China generated great concern from the public health community. The identification of ST11-CR-HvKP strain is expected to become a serious public health issue in China, considering the carbapenem resistance and virulence had converged in an epidemic clone. However, the underlying mechanism that enables its wide dissemination in China remains unclear.

Results: Here, we investigate the prevalence of carbapenemase-producing Enterobacteriaceae (CPE) carriage by inpatients in a teaching hospital over a 1-year period, to identify ST11-CR-HvKP reservoirs, and to understand the transmission of these pathogens across healthcare networks. We identified a high colonization prevalence of CPE (12.4\%) among inpatients with diarrhea. Correlations were detected between antibiotic exposure, surgical history, and being CPE positive. A genomic investigation of 65 CRKP isolates indicated a shared bacterial population among various wards. Maximum-likelihood phylogenetic tree demonstrated that these isolates were partitioned into three major clades. An analysis of the wzi locus revealed three different K types (KL105, KL47, and K64) among the ST11 isolates, indicating genetic diversity among these isolates. Our review of the cases showed that these patients had no contact with each other, indicating nosocomial transmission. Genetic and sequence mapping revealed complexity in the existence of virulence plasmids and resistance plasmids in the ST11-CRKP isolates. These data indicate that this process was more complicated than was earlier anticipated, as it may have involved multiple ST11 K. pneumoniae lineages and a variety of virulence plasmids.

Conclusions: Collectively, this work represents the first evidence of gut microbiota may act as the source of ST11-CR-HvKP isolate. Active surveillance approaches, particularly in ICUs based on the results of this study, should be implemented to combat the spread of ST11-CR-HvKP and to improve patient outcomes. Key words: gut microbiota; hypervirulent; KPC-2; reservoir; genomic characterization

\section{Introduction}

Carbapenem-resistant Klebsiella pneumoniae (CRKP), which is associated with high mortality rates of up to $50 \%$, has been identified by the World Health Organization as a critical priority organism [1,2]. Hypervirulent $K$. pneumoniae (HvKP) causes life-threatening infections, and the $r m p A / r m p A 2$ genes are associated with its pathogenicity [3]. Recently, a fatal outbreak of ST11 carbapenem-resistant, hypervirulent $K$. pneumoniae (ST11-CR-HvKP) in China generated great concern from the public health community [4]. The identification of this ST11-CR-HvKP strain demonstrated that carbapenem resistance and virulence had converged in an epidemic clone that could become a serious public health issue [5]. Subsequently, cases of ST11-CR-HvKP infection have been documented in diverse regions in China [6-8]. These reports suggest that this clone is widely spread in China; however, the underlying mechanism that enables its wide dissemination remains unclear. 
Intestinal tract colonization with carbapenemase-producing Enterobacteriaceae (CPE) may lead to subsequent nosocomial infections in at-risk patients [9-11]. Therefore, we hypothesized that inpatients might have a higher rate of CPE colonization and that intestinal carriage may represent a key factor underlying the epidemiology of ST11-CR-HvKP nosocomial infections in China. The aims of this study were to investigate the prevalence of CPE carriage of inpatients in a teaching hospital in China over a 1year period, to identify ST11-CR-HvKP reservoirs, and to understand the transmission of these pathogens across healthcare networks.

\section{Materials And Methods}

\section{Study design}

We conducted a prospective, observational cohort study of inpatients with acute diarrhea between February, 2016 and February, 2017 (Figure S1). Enterobacteriaceae isolates that were cultured from the first clinical samples of respective patients with diarrhea were collected. Duplicate isolates (the same species from the same patient within two weeks of the first positive culture) were excluded in this study. The study was performed at the First Affiliated Hospital of Zhejiang University (FAHZU), China, which is the largest tertiary teaching hospital in Zhejiang Province with 2,500 beds. Clinical data were extracted using centralized queries from clinical and medical record system used for all patients.

\section{Bacterial isolation and identification}

Fecal sample $(1.0 \mathrm{~g})$ were diluted in $5 \mathrm{ml}$ sterile Luria-Bertani liquid medium and cultured overnight at $37^{\circ} \mathrm{C}$. The enrichments were plated on MacConkey agar plates amended with $1 \mathrm{mg} / \mathrm{L}$ meropenem for $18-$ $24 \mathrm{~h}$ at $37^{\circ} \mathrm{C}$ to isolate potential carbapenem-resistant Enterobacteriaceae (CRE) strains [12]. Colonies with various morphologies were repeatedly streaked on the same medium to obtain pure isolates. Bacterial species were identified by matrix-assisted laser desorption ionization-time of flight massspectrometry (MALDI-TOF MS) and 16S rDNA sequencing using universal prokaryotic primers. CRE isolates were confirmed by susceptibility test with imipenem, ertapenem, and meropenem via broth microdilution method, and CPE isolates carbapenem resistance phenotype [13].

\section{Risk factor analysis}

To investigate the risk factors associated with fecal carriage of $\mathrm{CPE}$, carriers were compared with noncarriers in terms of exposure to the different variables studied. Categorical variables were expressed as percentages and were compared using the Chi-squared test or two-tailed Fisher exact test, as appropriate. Independent predictors for CPE were examined by logistic regression analysis. Variables with $\mathrm{P} \leq 0.2$ in the univariate analysis were included in a logistic regression model to identify variables with either negative or positive impact on the colonization of CPE in the acute diarrhea inpatients. The strength of associations was determined by calculating the odds ratio (OR) and $95 \%$ confidence intervals (Cls). 
Variables with a two-tailed P-value of $<0.05$ were considered statistically significant. All statistical analyses were performed using SPSS version 24.0.

\section{Antimicrobial susceptibility test (AST)}

The minimum inhibitory concentrations (MICs) of 14 antimicrobial agents (amikacin, aztreonam, cefotaxime, cefpirome, ceftazidime, ciprofloxacin, colistin, fosfomycin, gentamicin, imipenem, meropenem, piperacillin-tazobactam, tobramycin, and tigecycline) were determined by agar dilution methods, except for colistin and tigecycline, for which broth microdilution was used [14]. The results were interpreted using Clinics and Laboratory Standard Institute guideline (CLSI) breakpoints [13]. Standard reference strains of E. coli ATCC 25922and K. pneumoniae ATCC 700603 were used as quality control.

\section{Detection of targeted genes}

Genomic DNA was extracted from overnight cultures using the Qiagen Blood/Tissue Kit (Qiagen, Hilden, Germany). The presence of carbapenemase encoding genes (NDM, KPC, IMP and VIM) was detected via PCR and sequencing from each colony as described previously [14]. Detection of virulence-associated rmpA and rmpA2 genes in K. pneumoniae were conducted as described previously [15].

\section{Molecular typing of KPC-2-producing K. pneumoniae isolates}

Genetic relatedness of $K$. pneumoniae isolates was assessed by pulsed-field gel electrophoresis (PFGE) [16]. Briefly, DNA fragments were separated in $1 \%$ agarose (SeaKem Gold agarose, Lonza) at $14^{\circ} \mathrm{C}$ and 6 $\mathrm{V} / \mathrm{cm}$ and with alternating pulses at $120^{\circ}$ in a 2-40 s pulse time gradient for $22 \mathrm{~h}$ in $0.5 \times$ Tris-boric acidEDTA (TBE) buffer with CHEF apparatus (Bio-Rad, USA). Salmonella enterica serotype Braenderup H9812 was used as a size marker [17]. The dendrogram of PFGE patterns was constructed using BioNumerics v7.6 (Applied Maths, Sint-Martens-Latem, Belgium) with UPGMA clustering. Isolates that exhibited similarity cut-off $\geq 80 \%$ were considered a pulsotype.

\section{Illumina sequencing and sequence assembly}

To characterize the genetic features and resistome of KPC-2-producing K. pneumoniae isolates, wholegenome sequencing (WGS) was performed on all KPC-2-producing K. pneumoniae. Whole-cell DNA was extracted from overnight cultures using the Gentra Puregene Yeast/Bact Kit (Qiagen, Hilden, Germany). The harvested DNA was further detected in $1 \%(\mathrm{w} / \mathrm{v})$ agarose gels, and the concentration and purity of DNA were measured using NanoDrop 2000 UV-Vis Spectrophotometer (Thermo scientific, Waltham, MA, USA) and Qubit ${ }^{\circledR} 2.0$ Fluorometer (Thermo scientific, Waltham, MA, USA). DNA was stored at $-20^{\circ} \mathrm{C}$ until further processing. The sequencing library was produced by using Illumina Nextera XT Kit and sequenced on Illumina HiSeq X 10-PE150 platform (Illumina, San Diego, CA, USA). A-tailed, ligated to paired-end 
adaptors and PCR amplified with a $500 \mathrm{bp}$ insert and a mate-pair library with an insert size of $5 \mathrm{~kb}$ were used for the library construction at the Beijing Novogene Bioinformatics Technology Co., Ltd. PCR adapter reads and low-quality reads from the paired-end and mate-pair library were filtered by the step of quality control using Novogene pipeline. Paired reads were assembled into a number of scaffolds using Velvet 1.2.10 [18].

\section{Long-read Pacific Biosciences sequencing and assembly}

To further elucidate the genetic environment of $r m p A / r m p A 2$ carrying $K$. pneumoniae isolates, five randomly selected RmpA and RmpA2 positive isolates were subjected to the PacBio sequencing based on the size of $r m p A / r m p A 2$ carrying plasmids. The extracted DNA of isolates L39, L201, L388, L482, and L491 were sequenced by long-read single-molecule real-time (SMRT) sequencing technology using the PacBio RS II platform (Pacific Biosciences, Menlo Park, CA, USA). The extracted high-quality, doublestranded DNA $(10 \mu \mathrm{g})$ was sequenced using P6-C4 chemistry on the PacBio RS II instrumentation by using complexed 20-kb SMRTbell library. Unicycler was used for hybrid assembling the three bacterial genomes from a combination of Illumina short reads and Pacbio long reads [19]. We run Unicycler in three different modes (conservative, normal and bold) that alter its cutoff for minimum acceptable bridge quality to generate the best assemble results.

\section{Bioinformatics analysis and phylogenomic computations}

To determine the clonal lineages, the sequence types (STs) of KPC-2-producing K. pneumoniae isolates were determined by multilocus sequence typing (MLST) from WGS data in accordance with protocols described on the website (http://bigsdb.pasteur.fr/klebsiella/). ResFinder 3.1 was used to identify antimicrobial resistance genes [20]. Plasmid Finder 1.3 was used to identify the incompatibility type of the plasmids [21]. We further identified the virulence loci with the assembled genome sequences using BIGSdb Klebsiella genome database. We generated a heatmap of the virulence loci with Genesis software version 1.7.7. To find the core genes of the KPC-2-producing K. pneumoniae genomes, we used Prokka [22] and Roary [23]. Maximum likelihood-based phylogenetic reconstruction was performed with RAxML version 8.2.10 [24]. One hundred bootstrap replicates were evaluated to determine branch support. Maximum-likelihood phylogenetic tree based on core single nucleotide polymorphism (SNP) alignments was visualized with FastTree [25].

\section{Characterization of $r m p A / r m p A 2$ carrying plasmids}

S1 nuclease-pulsed-field gel electrophoresis (S1-PFGE) and Southern blot analysis were performed to estimate the size of the $r m p A / r m p A 2$ carrying plasmids [26]. The sequence of the plasmid carrying the rmpA/rmpA2 genes, were assembled using plasmidSPAdes from WGS data [27]. Annotation was performed using the RAST tool [28]. The sequences of represented plasmids was compared against other NCBI accessioned plasmids using BLAST and plotted by BLAST Ring Image Generator (BRIG) [29]. 


\section{Results}

During the study period, we sampled 811 non-duplicate stool samples from 443 inpatients and screened for the presence of CPE strains. Ultimately, $87 \mathrm{CPE}$ isolates from 55 patients were included in the study, and this frequency indicated a high CPE colonization prevalence (i.e., 12.4\%, 55/443) among inpatients with diarrhea (Figure S1). Of the 87 isolates, K. pneumoniae was the most prevalent species $(n=65)$, followed by Proteus mirabilis $(n=6)$, and Escherichia coli $(n=6)$ (Table S1). PCR and sequencing revealed that 77 isolates carried $b / a_{\mathrm{KPC}-2}$, five carried $b / a_{\mathrm{NDM}-5}$, two carried $b / a_{\mathrm{NDM}-1}$, two carried $b / a_{\mathrm{IMP}-}$ ${ }_{4}$, andone carried $b / a_{\mathrm{IMP}-26}$; furthermore, 49 isolates harbored extended-spectrum $\beta$-lactamase (ESBL) genes (Table S2). All of the CPE isolates were multidrug resistant (i.e., phenotypic resistance to three or more drug classes) (Figure S2). Among these CPE isolates, the highest resistance rate (99\%) was to imipenem, and most of the isolates were susceptible to colistin (92\%) and tigecycline (91\%).

Not surprisingly, most of the colonization cases were observed among patients from medical intensive care units (Table S1), since they typically experience prolonged hospital stays that result in exposure to potential risk factors. The patients came from 13 units: $49 \%$ (43/87) from intensive care unit (ICU), $9 \%$ from hepatobiliary and pancreatic surgery, $8 \%$ from emergency intensive care unit (EICU), and the remaining 33\% from ten other units. Among the patients, 42 (76\%) were male, and the median patient age was 64 years (range, 16-97). Interestingly, correlations were detected between antibiotic exposure (including linezolid, $\beta$-lactam- $\beta$-lactamase inhibitor, and carbapenems), surgical history, and being CPE positive (Table 1).

To better define the population structure of the 65 CRKPisolates, we further investigated their comprehensive molecular characteristics. Remarkably, an MLST analysis revealed that the $K$. pneumoniae isolates belonged to four STs, indicating low diversity (Figure 1 and Table S1). ST11 was the predominant ST $(58 / 65,89 \%)$, followed by ST37 $(n=5)$, ST15 $(n=1)$, and ST107 $(n=1)$. A notable feature was that patient P1 carried two STs: ST37, which was isolated from three samples, and ST11, which was isolated from eight samples, over the entire study period. Furthermore, ten patients in the study carried multiple isolates. Several major pulsotypes were found via PFGE analysis, which is consistent with the MLST results; however, genetic diversity was observed in the profiles (Figure S3). Roary matrix-based gene sequence analysis generated a large pan-genome of 10,355 gene clusters across 65 full genomes (Figure S4). A maximum-likelihood phylogenetic tree demonstrated that the 65 strains were partitioned into three major clades (Figure 1). Seven non-ST11 isolates were clustered into two separate clades, and the 58 ST11 isolates were grouped into three clusters, despite their high similarity. An analysis of the wzi locus revealed three different K types (KL105, KL47, and K64) among the ST11 isolates, indicating genetic diversity among these isolates. Of note, ST11 isolates were detected in patients from 12 wards. Our review of the cases showed that these patients had no contact with each other, making nosocomial transmission less likely.

A broad array of resistance genes associated with various antimicrobials was identified in the $K$. pneumoniae genomes (Figure 2). Isolates encoding carbapenemases $(44 / 65,68 \%)$ also harbored 
predicted ESBLs. Among these ESBLs, CTX-M- 65 was the most predominant cluster $(34 / 65,52 \%)$, followed by CTX-M-14 (5/65, 8\%). A virulence gene analysis revealed that the $K$. pneumoniae isolates carried genes for 45 known virulence factors (Figure 3). As expected, a high prevalence of biofilmencoding type 3 fimbriae clusters ( $m r k$ ) and yersiniabactin $(y b t)$-associated genes was observed in these isolates.

An in-silico analysis identified 14 rmpA- and 19 rmpA2-positive ST11 CRKP isolates. Interestingly, S1PFGE and Southern blot analyses clearly showed that at least four types of $r m p A / r m p A 2$-positive plasmids were carried by the ST11-CR-HvKP isolates, ranging in size from $\sim 146 \mathrm{~kb}$ to $218 \mathrm{~kb}$ (Figure 4). The coexistence of $r m p A$ and rmpA2 on the same plasmid was observed in 14 isolates. PacBio sequencing generated five complete $r m p A / r m p A 2$-encodinglncHI1B plasmid sequences that aligned well with the virulence plasmid pLVPK (AY378100), a $219 \mathrm{~kb}$ plasmid that harbors a different set of virulence genes, including iroBCDN, iuCABCD, rmpA, and $r m p A 2$ (Figure 5).

During the study period, we sampled 811 non-duplicate stool samples from 443 inpatients and screened for the presence of CPE strains. Ultimately, $87 \mathrm{CPE}$ isolates from 55 patients were included in the study, and this frequency indicated a high CPE colonization prevalence (i.e., 12.4\%, 55/443) among inpatients with diarrhea (Figure S1). Of the 87 isolates, K. pneumoniae was the most prevalent species $(n=65)$, followed by Proteus mirabilis $(n=6)$, and Escherichia coli $(n=6)$ (Table S1). PCR and sequencing revealed that 77 isolates carried $b / a_{\mathrm{KPC}-2}$, five carried $b / a_{\mathrm{NDM}-5}$, two carried $b / a_{\mathrm{NDM}-1}$, two carried $b / a_{\mathrm{IMP}-}$ 4 , andone carried $b / a_{1 \mathrm{MP}-26}$; furthermore, 49 isolates harbored extended-spectrum $\beta$-lactamase (ESBL) genes (Table S2). All of the CPE isolates were multidrug resistant (i.e., phenotypic resistance to three or more drug classes) (Figure S2). Among these CPE isolates, the highest resistance rate (99\%) was to imipenem, and most of the isolates were susceptible to colistin (92\%) and tigecycline (91\%).

Not surprisingly, most of the colonization cases were observed among patients from medical intensive care units (Table S1), since they typically experience prolonged hospital stays that result in exposure to potential risk factors. The patients came from 13 units: $49 \%$ (43/87) from intensive care unit (ICU), $9 \%$ from hepatobiliary and pancreatic surgery, $8 \%$ from emergency intensive care unit (EICU), and the remaining 33\% from ten other units. Among the patients, 42 (76\%) were male, and the median patient age was 64 years (range, 16-97). Interestingly, correlations were detected between antibiotic exposure (including linezolid, $\beta$-lactam- $\beta$-lactamase inhibitor, and carbapenems), surgical history, and being CPE positive (Table 1).

To better define the population structure of the 65 CRKPisolates, we further investigated their comprehensive molecular characteristics. Remarkably, an MLST analysis revealed that the $K$. pneumoniae isolates belonged to four STs, indicating low diversity (Figure 1 and Table S1). ST11 was the predominant ST $(58 / 65,89 \%)$, followed by ST37 $(n=5)$, ST15 $(n=1)$, and ST107 $(n=1)$. A notable feature was that patient P1 carried two STs: ST37, which was isolated from three samples, and ST11, which was isolated from eight samples, over the entire study period. Furthermore, ten patients in the study carried multiple isolates. Several major pulsotypes were found via PFGE analysis, which is 
consistent with the MLST results; however, genetic diversity was observed in the profiles (Figure S3). Roary matrix-based gene sequence analysis generated a large pan-genome of 10,355 gene clusters across 65 full genomes (Figure S4). A maximum-likelihood phylogenetic tree demonstrated that the 65 strains were partitioned into three major clades (Figure 1). Seven non-ST11 isolates were clustered into two separate clades, and the 58 ST11 isolates were grouped into three clusters, despite their high similarity. An analysis of the wzi locus revealed three different $\mathrm{K}$ types (KL105, KL47, and K64) among the ST11 isolates, indicating genetic diversity among these isolates. Of note, ST11 isolates were detected in patients from 12 wards. Our review of the cases showed that these patients had no contact with each other, making nosocomial transmission less likely.

A broad array of resistance genes associated with various antimicrobials was identified in the $K$. pneumoniae genomes (Figure 2). Isolates encoding carbapenemases $(44 / 65,68 \%)$ also harbored predicted ESBLs. Among these ESBLs, CTX-M- 65 was the most predominant cluster $(34 / 65,52 \%)$, followed by CTX-M-14 (5/65, 8\%). A virulence gene analysis revealed that the $K$. pneumoniae isolates carried genes for 45 known virulence factors (Figure 3 ). As expected, a high prevalence of biofilmencoding type 3 fimbriae clusters ( $m r k$ ) and yersiniabactin ( $y b t)$-associated genes was observed in these isolates.

An in-silico analysis identified 14 rmpA-and 19 rmpA2-positive ST11 CRKP isolates. Interestingly, S1PFGE and Southern blot analyses clearly showed that at least four types of $r m p A / r m p A 2$-positive plasmids were carried by the ST11-CR-HvKP isolates, ranging in size from $\sim 146 \mathrm{~kb}$ to $\sim 218 \mathrm{~kb}$ (Figure 4). The coexistence of $r m p A$ and $r m p A 2$ on the same plasmid was observed in 14 isolates. PacBio sequencing generated five complete $r m p A / r m p A 2$-encodinglncHI1B plasmid sequences that aligned well with the virulence plasmid pLVPK (AY378100), a $219 \mathrm{~kb}$ plasmid that harbors a different set of virulence genes, including iroBCDN, iucABCD, rmpA, and rmpA2 (Figure 5).

\section{Discussion}

The first isolation of ST11-CRKP in China occurred at FAHZU in 2004 [30]. Over the last few decades, China has witnessed the emergence and subsequent rapid increase in the number of ST11-CRKP nosocomial infections [31]. However, the role of the gut microbiota in the rapid spread of ST11-CRKP has not been well elucidated. Our experimental evidence, as well as the retrieved clinical data, revealed a disturbing carriage rate of ST11-CRKP (8.6\%) among hospitalized patients with acute diarrhea, particularly in ICU wards. Our findings suggest that the gut microbiota could be a major reservoir of ST11CR-HvKP. Active surveillance approaches, particularly in ICUs based on the results of this study, should be implemented to combat the spread of ST11-CRKP and to improve patient outcomes.

To our knowledge, an association between linezolid exposure CPE colonization in these types of patient populations has not been reported. The lack of such an association might be due to the wide use of linezolid, which eliminates Gram-positive bacteria and disrupts the gut microflora, thus promoting gut colonization by Enterobacteriaceae, especially CPE strains. 
A previous investigation speculated that the emergence of ST11-CR-HVKP occurred due to a single genetic event in which a pLVPK-like virulence plasmid was acquired by an ST11-CRKP strain that presumably carried a common plasmid such as pKPC-CR-HvKP4 [4]. The observed complexity in the existence of virulence plasmids and resistance plasmids in the ST11-CRKP isolates indicates that this process was more complicated than was earlier anticipated, as it may have involved multiple ST11 $\mathrm{K}$. pneumoniae lineages and a variety of virulence plasmids.

This study has several limitations. First, our study is limited by its single-institution design and 1-year study period; therefore, the findings of this study should be interpreted with caution and might not be generalizable to other hospitals. Second, although we identified 19 fecal ST11-CR-HvKP colonization isolates, none of the colonized patients had ST11-CR-HvKP infections. A previous investigation described the high prevalence and mortality rate of ST11-CR-HvKP meningitis in our hospital [8], and K64 was also the most common serotype detected in this study. Future investigations addressing the transition from ST11-CR-HvKP carriage to infection in high-risk patients will be of great significance.

\section{Conclusions}

In conclusion, our genomic epidemiologic investigation demonstrated that the gut microbiota of hospitalized patients served as the reservoir of CPE isolates, particularly ST11-CR-HvKP. Because of the serious clinical outcomes of CRKP infections, more information is needed to understand the potential risk of ST11-CR-HvKP spreading by inpatients and to develop measures for surveillance or control of these risks.

\section{Abbreviations}

ST11-CR-HvKP: ST11 carbapenem-resistant, hypervirulent K. pneumoniae; CRE: carbapenem-resistant Enterobacteriaceae; CPE: carbapenemase-producing Enterobacteriaceae; CRKP: carbapenem-resistant $K$. pneumoniae; MALDI-TOF MS: matrix-assisted laser desorption ionization-time of flight mass spectrometry; MICs: minimum inhibitory concentrations; CLSI: Clinics and Laboratory Standard Institute guideline; MLST: multilocus sequence typing; SNP: single nucleotide polymorphism; S1-PFGE: S1 nuclease-pulsed field gel electrophoresis.

\section{Declarations}

\section{Ethics approval and consent to participate}

Informed consent was obtained from all patients according to the ethical protocol approved by the Ethics Committee of FAHZU (no. 2018-1031)

\section{Consent for publication}


Not applicable.

\section{Availability of data and material}

The whole genome sequences of 65 CRKP have been deposited in GenBank under the BioProject numbers PRJNA390758.

\section{Competing interests}

The authors declare that they have no competing interests.

\section{Funding}

This study was partially funded by grants from the National Key Research and Development Program of China (Nos. 2016YFD0501105 and 2017YFC1200203); the Mega-projects of Science Research of China (Nos. 2018ZX10733402-004 and 2018ZX10712001-005); the National Natural Science Foundation of China (Nos. 81741098 and 81711530049); the Zhejiang Provincial Key Research and Development Program (No. 2015C03032); the Zhejiang Provincial Natural Science Foundation of China (No. LY17H190003).

\section{Authors' contributions}

$B Z, Y X$, and LL designed this study. TL and YC collected the fecal samples and screened the isolates. HX performed the microbiological and molecular experiments. $\mathrm{LG}, \mathrm{XY}, \mathrm{CH}, \mathrm{SZ}, \mathrm{HH}$, and PS analyzed the data. $B Z$ wrote the manuscript and $Y X$, and LL revised it. All authors read and approved the final manuscript.

\section{Acknowledgements}

We thank the personnel of the clinical microbiology laboratory at FAHZU for assistance with collecting samples.

\section{Additional Files}

Additional file 1: Table S1. Carbapenemase-producing Enterobacteriaceae isolates recovered from stool specimens.

Additional file 2: Table S2. Summary of the carbapenemase-producing Enterobacteriaceae (CPE) isolates and the $\beta$-lactamases encoded by the CPE isolates. 
Additional file 3: Figure S1. Flowchart of the included cases and the analyses performed. Abbreviations: $\mathrm{CPE}$, carbapenemase-producing Enterobacteriaceae; ARGs, antimicrobial resistance genes.

Additional file 4: Figure S2. Antimicrobial susceptibility profiles of $87 \mathrm{CPE}$ isolates. The MICs were determined via an agar dilution method for all antibiotics except for colistin and tigecycline, for which a broth microdilution method was used. Unless otherwise specified, the susceptibility tests were interpreted according to the 2017 Clinical Laboratory Standards Institute (CLSI) criteria. Pink indicates resistance, blue indicates intermediate, and yellow indicates sensitivity.

Additional file 5: Figure S4. A pan-genome analysis of 65 CRKP strains, performed using Roary. The blue bar indicates the pan-genome of CRKP, including the 10,3554 annotated genes detected among the genomes analyzed in this study.

Additional file 6: Figure S3. A PFGE dendrogram generated using the Bionumerics software showing the genetic relationships between $65 \mathrm{CRKP}$ isolates. The PFGE pattern analysis demonstrated that 61 of the 65 CRKP isolates could be classified into nine pulsotypes: $A(n=2), B(n=20), C(n=9), D(n=8), E(n=$ 8), $F(n=2), G(n=2), H(n=5)$, and I $(n=5)$. Four isolates appeared to be singletons. The dashed line corresponds to $80 \%$ as the cutoff for a close genetic relationship.

\section{References}

1.Giacobbe DR, Del Bono V, Trecarichi EM, De Rosa FG, Giannella M, Bassetti M, Bartoloni A, Losito AR, Corcione S, Bartoletti $\mathrm{M}$ et al: Risk factors for bloodstream infections due to colistin-resistant KPCproducing Klebsiella pneumoniae: results from a multicenter case-control-control study. Clin Microbiol Infect 2015, 21(12):1106 e1101-1108.

2.Pecora ND, Li N, Allard M, Li C, Albano E, Delaney M, Dubois A, Onderdonk AB, Bry L: Genomically Informed Surveillance for Carbapenem-Resistant Enterobacteriaceae in a Health Care System. MBio 2015, 6(4): 001030.

3.Russo TA, Marr CM: Hypervirulent Klebsiella pneumoniae. Clin Microbiol Rev 2019, 32(3).

4.Gu D, Dong N, Zheng Z, Lin D, Huang M, Wang L, Chan EW, Shu L, Yu J, Zhang R et al: A fatal outbreak of ST11 carbapenem-resistant hypervirulent Klebsiella pneumoniae in a Chinese hospital: a molecular epidemiological study. Lancet Infect Dis 2018, 18(1):37-46.

5.Chen L, Kreiswirth BN: Convergence of carbapenem-resistance and hypervirulence in Klebsiella pneumoniae. Lancet Infect Dis 2018, 18(1):2-3.

6.Wong MHY, Shum HP, Chen JHK, Man MY, Wu A, Chan EW, Yuen KY, Chen S: Emergence of carbapenemresistant hypervirulent Klebsiella pneumoniae. Lancet Infect Dis 2018, 18(1):24. 
7.Yao H, Qin S, Chen S, Shen J, Du XD: Emergence of carbapenem-resistant hypervirulent Klebsiella pneumoniae. Lancet Infect Dis 2018, 18(1):25.

8.Xu M, Fu Y, Fang Y, Xu H, Kong H, Liu Y, Chen Y, Li L: High prevalence of KPC-2-producing hypervirulent Klebsiella pneumoniae causing meningitis in Eastern China. Infect Drug Resist 2019, 12:641-653.

9.Viau R, Frank KM, Jacobs MR, Wilson B, Kaye K, Donskey CJ, Perez F, Endimiani A, Bonomo RA: Intestinal Carriage of Carbapenemase-Producing Organisms: Current Status of Surveillance Methods. Clin Microbiol Rev 2016, 29(1):1-27.

10.Gorrie CL, Mirceta M, Wick RR, Edwards DJ, Thomson NR, Strugnell RA, Pratt NF, Garlick JS, Watson KM, Pilcher DV et al: Gastrointestinal Carriage Is a Major Reservoir of Klebsiella pneumoniae Infection in Intensive Care Patients. Clin Infect Dis 2017, 65(2):208-215.

11.Shimasaki T, Seekatz A, Bassis C, Rhee Y, Yelin RD, Fogg L, Dangana T, Cisneros EC, Weinstein RA, Okamoto K et al: Increased Relative Abundance of Klebsiella pneumoniae Carbapenemase-producing Klebsiella pneumoniae Within the Gut Microbiota Is Associated With Risk of Bloodstream Infection in Long-term Acute Care Hospital Patients. Clin Infect Dis 2019, 68(12):2053-2059.

12.Zheng B, Lv T, Xu H, Yu X, Chen Y, Li J, Huang C, Guo L, Zhang J, Jiang X et al: Discovery and characterisation of an escherichia coli ST206 strain producing NDM-5 and MCR-1 from a patient with acute diarrhoea in China. Int J Antimicrob Agents 2018, 51(2):273-275.

13.CLSI: Performance Standards for Antimicrobial Susceptibility Testing. 27th ed. CLSI supplement M100. Wayne, PA, USA: Clinical and Laboratory Standards Institute. 2017.

14.Zheng B, Zhang J, Ji J, Fang Y, Shen P, Ying C, Lv J, Xiao Y, Li L: Emergence of Raoultella ornithinolytica coproducing IMP-4 and KPC-2 carbapenemases in China. Antimicrob Agents Chemother 2015, 59(11):7086-7089.

15.Choi MJ, Ko KS: Loss of hypermucoviscosity and increased fitness cost in colistin-resistant Klebsiella pneumoniae sequence type 23 strains. Antimicrob Agents Chemother 2015, 59(11):6763-6773.

16.Xu H, Wang X, Yu X, Zhang J, Guo L, Huang C, Jiang X, Li X, Feng Y, Zheng B: First detection and genomics analysis of KPC-2-producing Citrobacter isolates from river sediments. Environ Pollut 2018, 235:931-937.

17.Shen P, Wei Z, Jiang Y, Du X, Ji S, Yu Y, Li L: Novel genetic environment of the carbapenem-hydrolyzing beta-lactamase KPC-2 among Enterobacteriaceae in China. Antimicrob Agents Chemother 2009, 53(10):4333-4338.

18.Zerbino DR, Birney E: Velvet: algorithms for de novo short read assembly using de Bruijn graphs. Genome Res 2008, 18(5):821-829. 
19.Wick RR, Judd LM, Gorrie CL, Holt KE: Unicycler: Resolving bacterial genome assemblies from short and long sequencing reads. PLoS Comput Biol 2017, 13(6):e1005595.

20.Zankari E, Hasman H, Cosentino S, Vestergaard M, Rasmussen S, Lund O, Aarestrup FM, Larsen MV: Identification of acquired antimicrobial resistance genes. J Antimicrob Chemother 2012, 67(11):26402644.

21.Carattoli A, Zankari E, Garcia-Fernandez A, Voldby Larsen M, Lund O, Villa L, Moller Aarestrup F, Hasman $\mathrm{H}$ : In silico detection and typing of plasmids using PlasmidFinder and plasmid multilocus sequence typing. Antimicrob Agents Chemother 2014, 58(7):3895-3903.

22.Seemann T: Prokka: rapid prokaryotic genome annotation. Bioinformatics 2014, 30(14):2068-2069.

23.Page AJ, Cummins CA, Hunt M, Wong VK, Reuter S, Holden MT, Fookes M, Falush D, Keane JA, Parkhill J: Roary: rapid large-scale prokaryote pan genome analysis. Bioinformatics 2015, 31(22):3691-3693.

24.Stamatakis A: RAXML version 8: a tool for phylogenetic analysis and post-analysis of large phylogenies. Bioinformatics 2014, 30(9):1312-1313.

25.Price MN, Dehal PS, Arkin AP: FastTree: computing large minimum evolution trees with profiles instead of a distance matrix. Mol Biol Evol 2009, 26(7):1641-1650.

26.Zheng B, Dong H, Xu H, Lv J, Zhang J, Jiang X, Du Y, Xiao Y, Li L: Coexistence of MCR-1 and NDM-1 in Clinical Escherichia coli Isolates. Clin Infect Dis 2016, 63(10):1393-1395.

27.Antipov D, Hartwick N, Shen M, Raiko M, Lapidus A, Pevzner PA: plasmidSPAdes: assembling plasmids from whole genome sequencing data. Bioinformatics 2016, 32(22):3380-3387.

28. Overbeek R, Olson R, Pusch GD, Olsen GJ, Davis JJ, Disz T, Edwards RA, Gerdes S, Parrello B, Shukla M et al: The SEED and the Rapid Annotation of microbial genomes using Subsystems Technology (RAST). Nucleic Acids Res 2014, 42(Database issue):D206-214.

29.Alikhan NF, Petty NK, Ben Zakour NL, Beatson SA: BLAST Ring Image Generator (BRIG): simple prokaryote genome comparisons. BMC Genomics 2011, 12:402.

30.Wei ZQ, Du XX, Yu YS, Shen P, Chen YG, Li LJ: Plasmid-mediated KPC-2 in a Klebsiella pneumoniae isolate from China. Antimicrob Agents Chemother 2007, 51(2):763-765.

31.Zhang R, Liu L, Zhou H, Chan EW, Li J, Fang Y, Li Y, Liao K, Chen S: Nationwide Surveillance of Clinical Carbapenem-resistant Enterobacteriaceae (CRE) Strains in China. EBioMedicine 2017.

\section{Tables}


Table 1. Characteristics of the study participants and logistic regression analysis of the carbapenemaseproducing Enterobacteriaceae (CPE) strains isolated from stool samples from these inpatients.

\begin{tabular}{|c|c|c|c|c|c|}
\hline Variable & $\begin{array}{l}\text { CPE-positive } \\
\text { group }\end{array}$ & $\begin{array}{l}\text { CPE-negative } \\
\text { group }\end{array}$ & $\mathrm{P}^{\mathrm{a}}$ & $\mathrm{P}^{\mathrm{b}}$ & OR $(95 \% \mathrm{CI})$ \\
\hline No. of patients (\%) & $55(12)$ & $388(88)$ & & & \\
\hline Male gender (\%) & $42(76)$ & $255(66)$ & 0.116 & 0.717 & $\begin{array}{l}1.143(0.556- \\
2.350)\end{array}$ \\
\hline Age $^{\mathrm{C}}$ [median(range) $]$ & $64(16-97)$ & $55(13-99)$ & $0.047^{\mathrm{C}}$ & 0.677 & $\begin{array}{l}1.146(0.603- \\
2.179)\end{array}$ \\
\hline \multicolumn{6}{|l|}{ No. (\%) with } \\
\hline Surgery & $16(29)$ & $54(14)$ & 0.004 & 0.047 & $\begin{array}{l}2.078(1.008- \\
4.282)\end{array}$ \\
\hline Abdominal pain & $13(24)$ & $130(34)$ & 0.14 & 0.578 & $\begin{array}{l}0.818(0.402- \\
1.665)\end{array}$ \\
\hline Fever & $43(78)$ & $244(63)$ & 0.026 & 0.595 & $\begin{array}{l}1.257(0.541- \\
2.919)\end{array}$ \\
\hline $\begin{array}{l}\text { No. (\%) of hospital-acquired } \\
\text { infections }\end{array}$ & $32(58)$ & $199(51)$ & 0.124 & 0.958 & $\begin{array}{l}0.97(0.319- \\
2.951)\end{array}$ \\
\hline \multicolumn{6}{|l|}{$\begin{array}{l}\text { No. (\%) receiving antibiotic } \\
\text { therapy }\end{array}$} \\
\hline Cephalosporins & $15(27)$ & 73 (19) & 0.141 & 0.349 & $\begin{array}{l}1.4(0.692- \\
2.833)\end{array}$ \\
\hline Carbapenems & $42(76)$ & $221(57)$ & 0.006 & 0.156 & $\begin{array}{l}1.739(0.81- \\
3.732)\end{array}$ \\
\hline Cephamycin & $0(0)$ & $15(4)$ & 0.234 & - & - \\
\hline Aminoglycosides & $5(9)$ & $38(10)$ & 0.869 & - & - \\
\hline Macrolides & $3(6)$ & $18(5)$ & 0.735 & - & - \\
\hline Glycopeptides & $20(36)$ & $108(28)$ & 0.192 & 0.674 & $\begin{array}{l}1.151(0.598- \\
2.218)\end{array}$ \\
\hline Oral vancomycin & $8(15)$ & $32(8)$ & 0.127 & 0.705 & $\begin{array}{l}1.196(0.473- \\
3.021)\end{array}$ \\
\hline Fosfomycin & $1(2)$ & $23(6)$ & 0.339 & - & - \\
\hline Linezolid & $23(42)$ & $56(14)$ & $<0.001$ & 0.002 & $\begin{array}{l}2.871(1.461- \\
5.642)\end{array}$ \\
\hline Quinolone & $16(29)$ & $160(41)$ & 0.085 & 0.174 & $\begin{array}{l}0.616(0.306- \\
1.238)\end{array}$ \\
\hline Sulfamethoxazole & $4(7)$ & $68(18)$ & 0.054 & 0.457 & $\begin{array}{l}0.637(0.194- \\
2.089)\end{array}$ \\
\hline$\beta$-lactam $/ \beta$-lactamase inhibitor & $45(82)$ & $249(64)$ & 0.01 & 0.039 & $\begin{array}{l}2.276(1.043- \\
4.969)\end{array}$ \\
\hline Tigecycline & $15(27)$ & $63(16)$ & 0.044 & 0.67 & $\begin{array}{l}0.849(0.401- \\
1.798)\end{array}$ \\
\hline
\end{tabular}

${ }^{a}$ Univariate analysis; categorical variables were compared using $\mathrm{x}^{2}$ test

${ }^{b}$ Multivariate analysis of variables $(\mathrm{P} \leq 0.20)$ in univariate analysis was performed using stepwise backward logistic regression. Odds ratios (ORs) and their 95\% confidence intervals (CIs) were calculated.

${ }^{\mathrm{c}} \mathrm{P}$ values were calculated based on a comparison between ages $\geq 60$ and $<60$ using $\mathrm{x}^{2}$ test. 


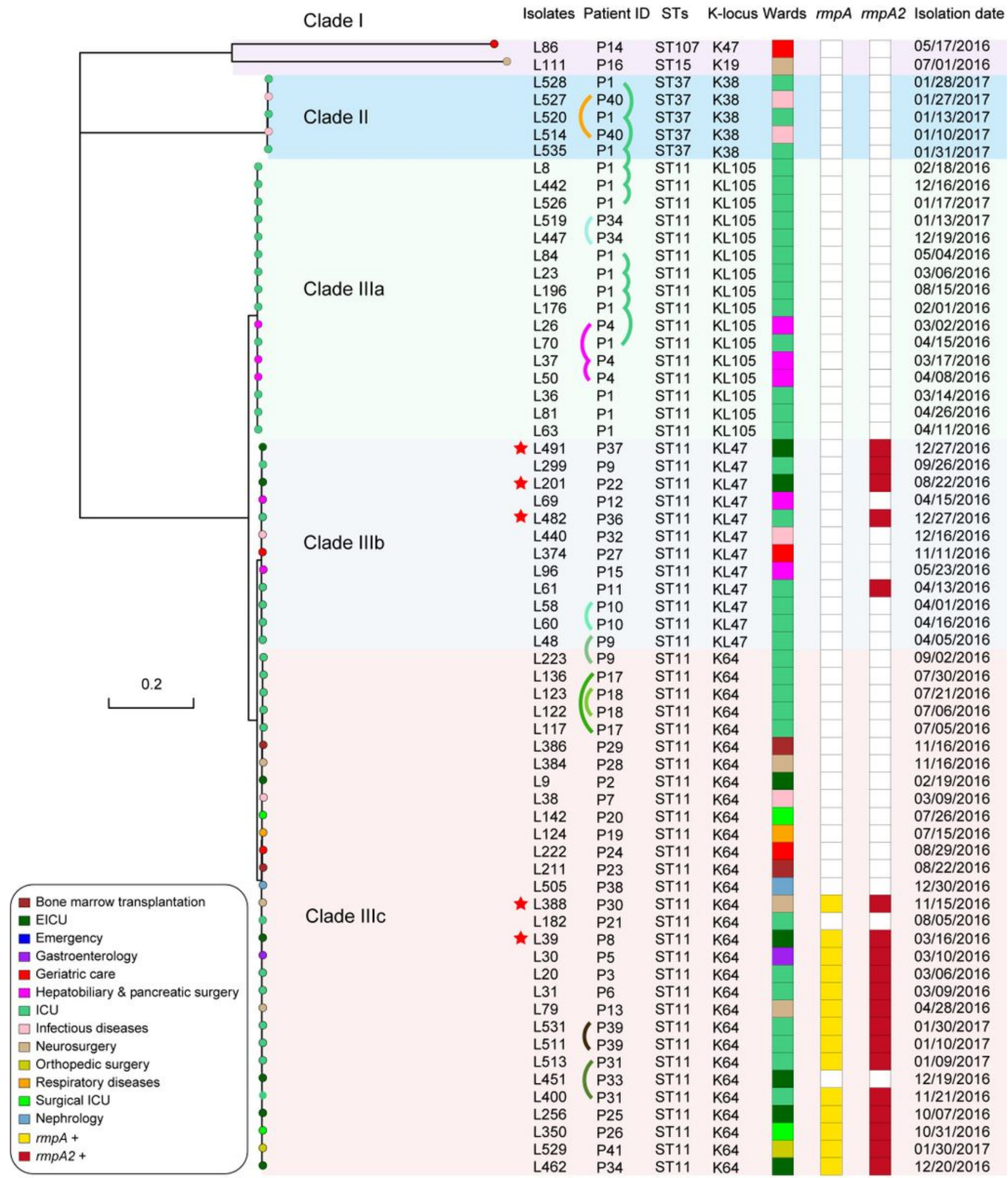

\section{Figure 1}

Core genome phylogeny for the CRKP isolates, including the associated multilocus sequence typing result, capsule locus genotypes, wards, virulence genes present, and isolation date. This maximumlikelihood phylogeny tree is based on single-nucleotide polymorphisms (SNPs) in the core genomes of 65 
CRKP isolates. The scale bar indicates nucleotide divergence. The origins of the isolates are shown in different colors. Genome clusters are shaded with different colors. The presence of rmpA and rmpA2 are indicated by yellow and red shapes, respectively. The curved lines indicate isolates from the same patient. The asterisks in red indicate isolates were conducted with PacBio sequencing.

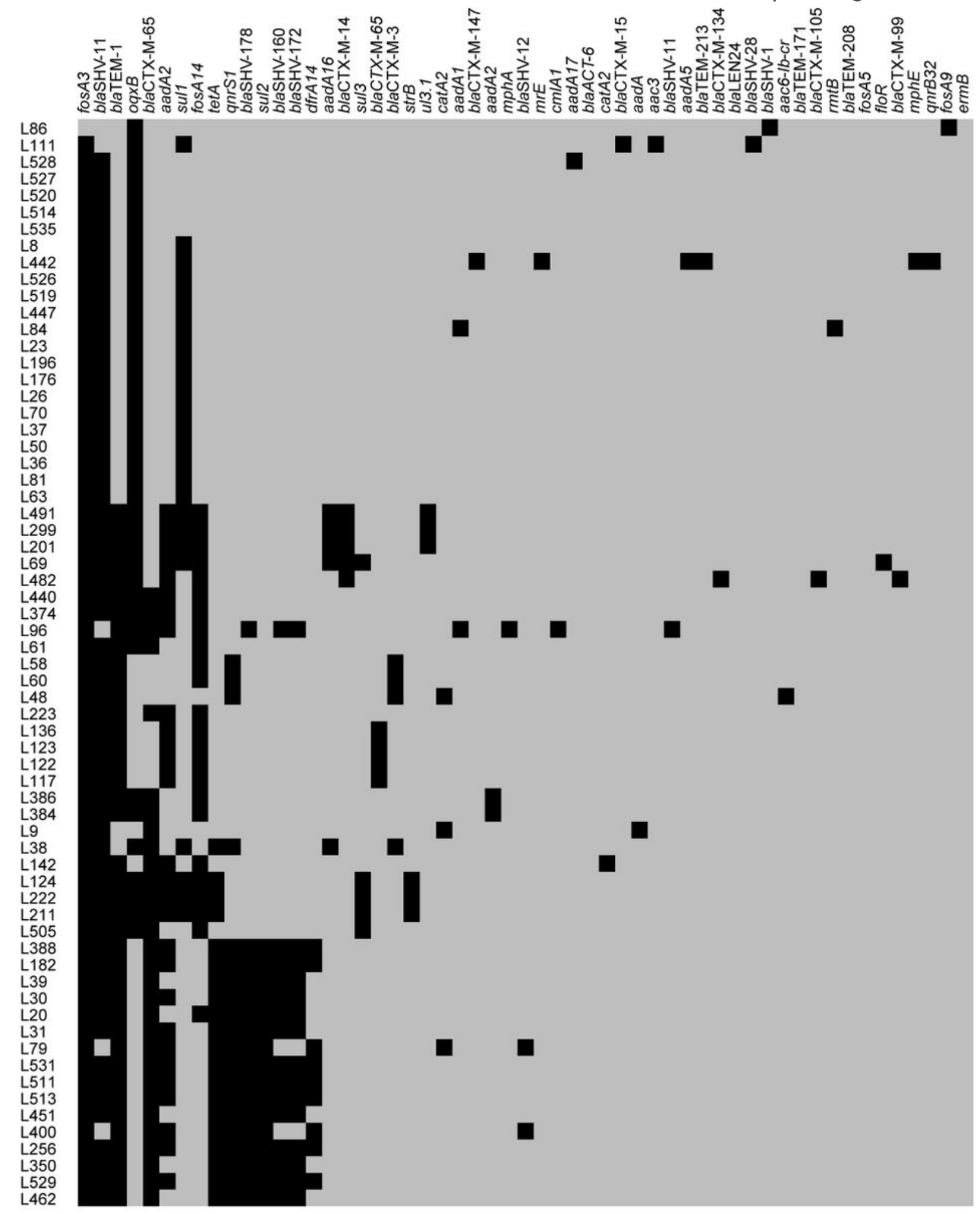

Figure 2 
Antimicrobial resistance genes identified in the genomes of CRKP isolates from WGS data. The antimicrobial resistance genes are shown on the right side with their presence indicated by black shapes.

ybts

kfuC

kfuA

kfuB

$c / b R$

$c l b Q$

$c l b P$

$c l b O$

$c / b N$

$c l b M$

$c l b L$

$c \mid b l$

$\mathrm{clbH}$

clbG

$c l b F$

clbE

$C l b D$

$c l b C$

CIbA

$C l b B$

imp1

mpA

iucA

iucB

iutA

mpA2

iucC

iucD

$i p 2$

$m k \mathrm{kH}$

$m \pi \mathrm{kJ}$

$m k l$

$m k \mathrm{~F}$

$m k \mathrm{k}$

$m k C$

$m k A$

$m k B$

$y b t x$

$y b t Q$

ybte

$y b t P$

ybtT

ybtU

fyuA

$y b t A$

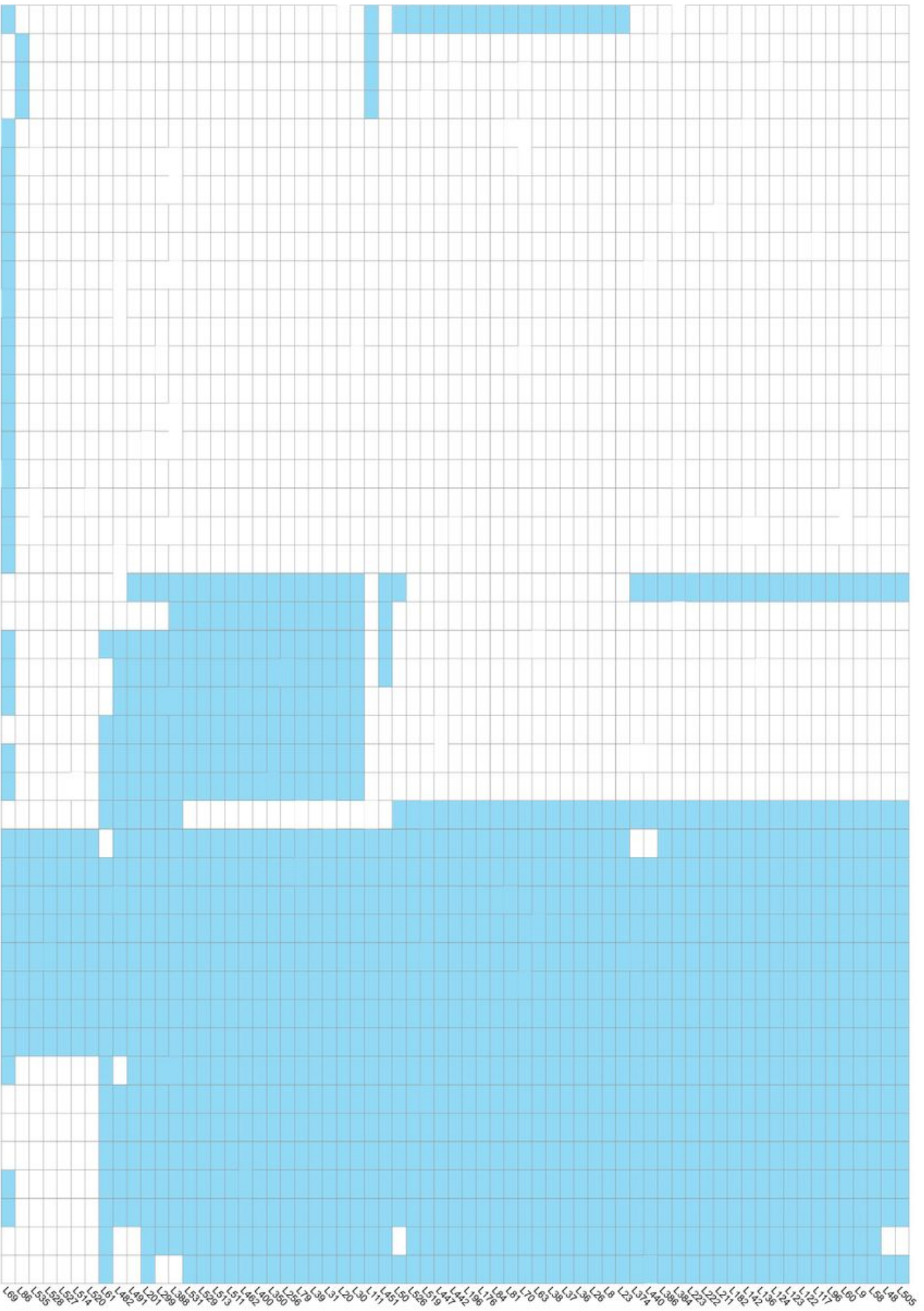

\section{Figure 3}

Distribution of virulence-associated genes in Klebsiella pneumoniae strains. Heatmaps were generated by aligning the draft genome sequence of each isolate to the BIGSdb-Kp database. The presence of 
virulence genes in a specific genome is represented by a blue box, and the absence of virulence genes is represented by a cream-colored box. Virulence factors are shown on the left side.

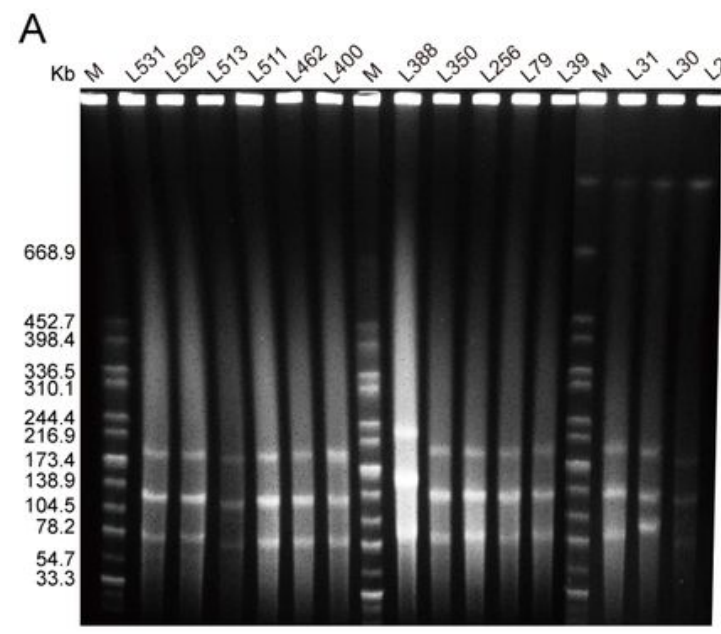

B
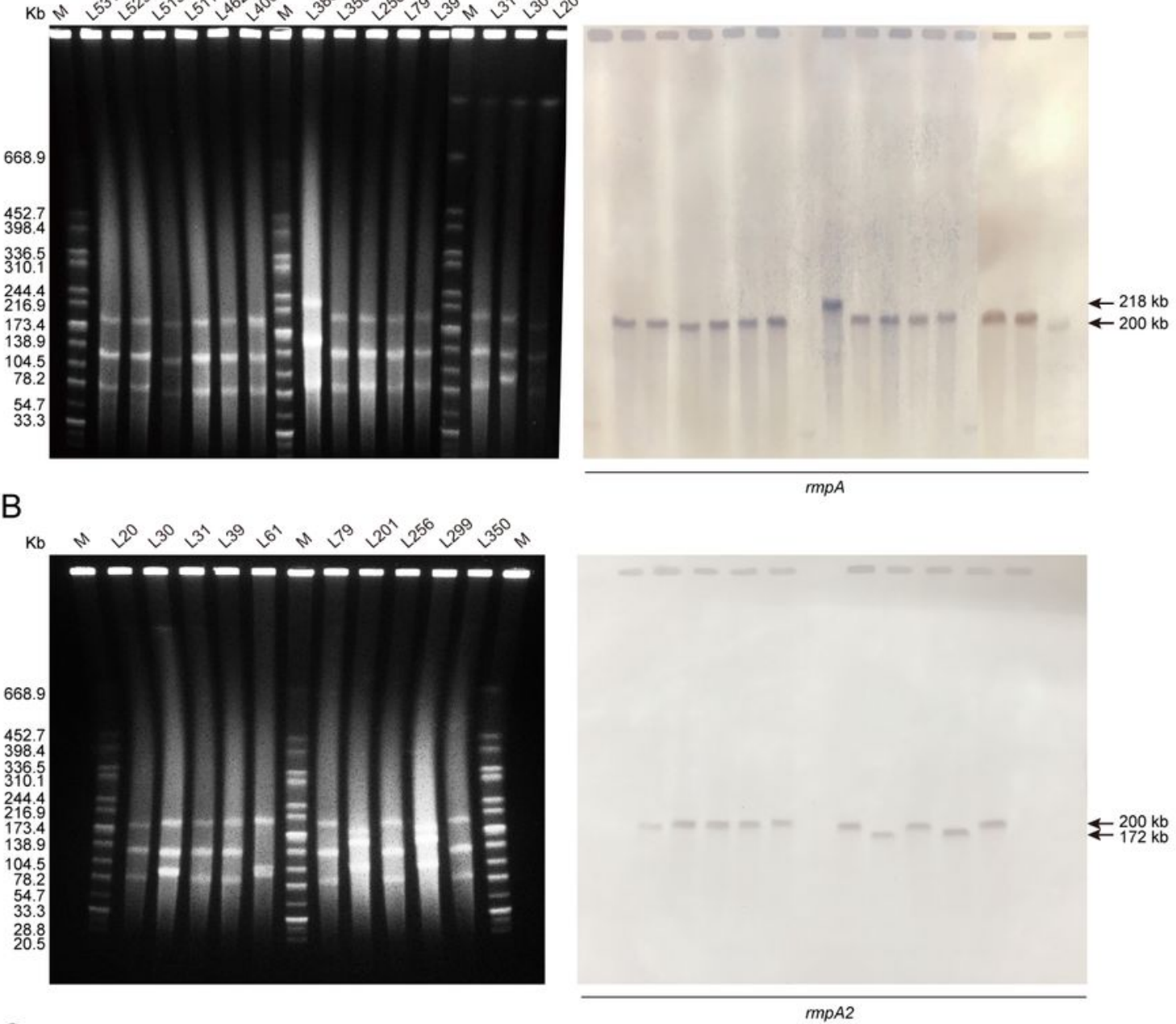

C
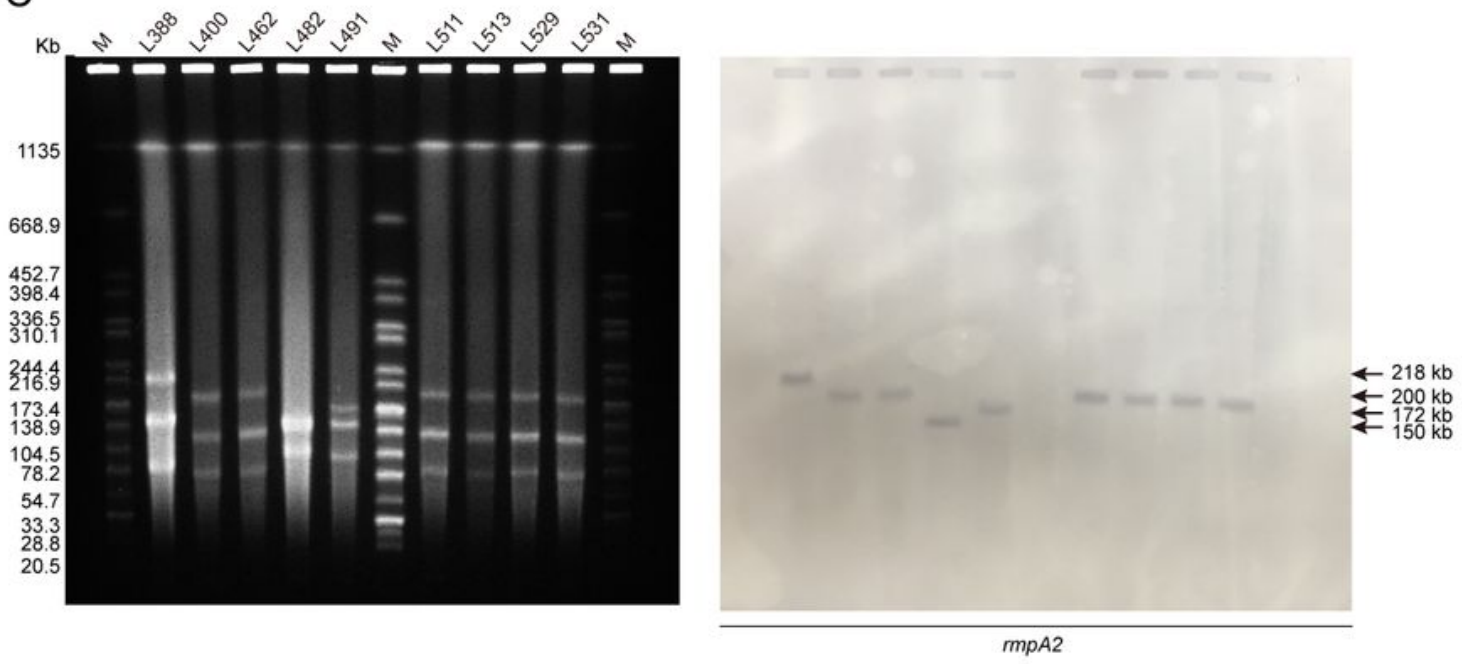

Figure 4

Plasmid analysis of the ST11 CR-HvKP isolates via S1-PFGE and Southern blotting using rmpA and rmpA2 probes. The arrows indicate the locations of the virulence plasmids. S1-PFGE revealed that most of the K. pneumoniae strains harbored three plasmids. 
A

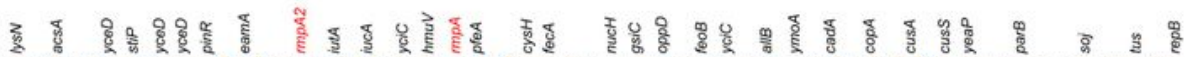

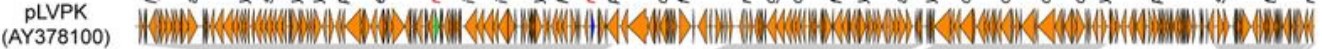

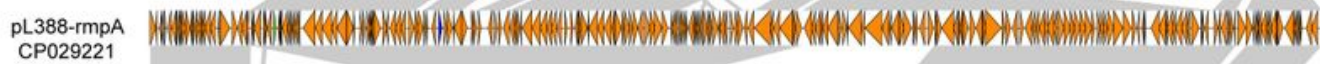

CP033955

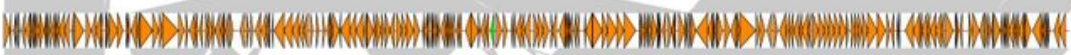

pL201-rmpaz
CP029217

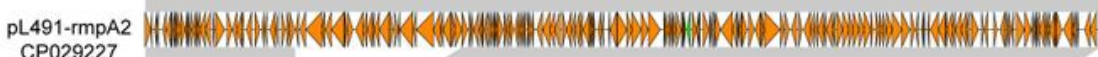

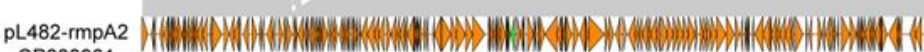

CP033961 \%

B

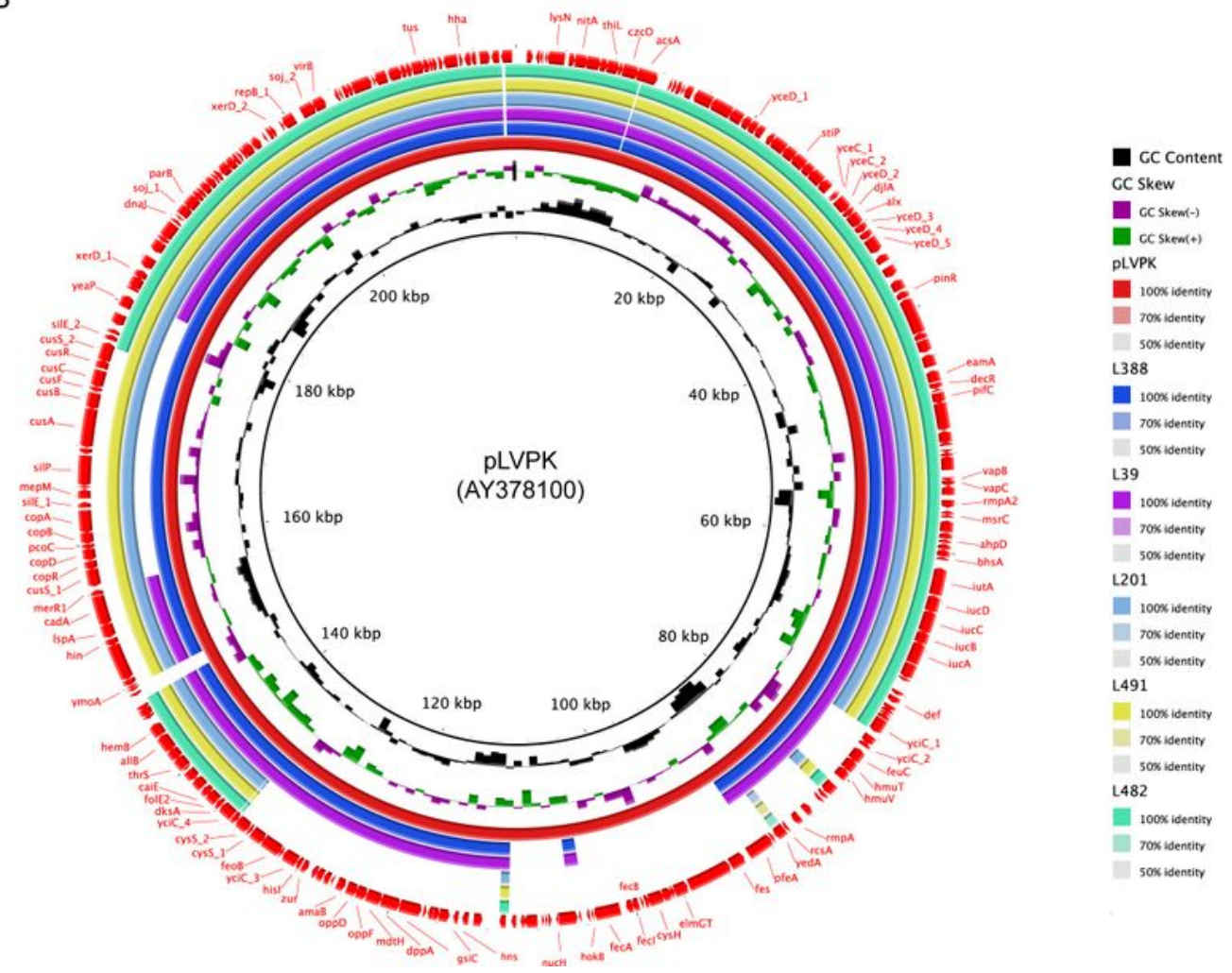

Figure 5

Alignment of rmpA/rmpA2-harbouring plasmids recovered in this work. The circular map was generated with the BLAST Ring Image Generator.

\section{Supplementary Files}


This is a list of supplementary files associated with this preprint. Click to download.

- Additonalfiles.docx 